\title{
Validity and clinical utility of a Sinhalese version of the Abnormal Involuntary Movement Scale (AIMS)
}

\author{
A K A B Baminiwatta, M L Harshini, T R Bandara, T Gunesekara, K M N Perera, K A L A \\ Kuruppuarachchi, A Hapangama
}

\section{Abstract \\ Introduction}

Tardive dyskinesia (TD) is a movement disorder caused by long-term treatment with dopamine antagonists such as antipsychotics. As there is no medication universally effective for TD, prevention is important. We propose that non-medical clinicians working in psychiatry can also be trained and involved in a screening programme for TD. To facilitate such a programme, validation of a standard tool translated to Sinhala is a need.

Aims

To translate and validate the Abnormal Involuntary Movement Scale (AIMS) into Sinhala.

\section{Methods}

Translation and pilot testing followed standard guidelines. The translated version was administered by five raters (two registrars in psychiatry, a medical officer, a community psychiatry nurse (CPN) and a psychiatric social worker) amongst patients treated with antipsychotic medication for a minimum of one year. The goldstandard diagnosis was the Diagnostic and Statistical Manual (DSM)-5 criteria, applied by a psychiatrist.

\section{Results}

Of the 137 patients screened $(53.3 \%$ male; mean age $=$ 49 years), the percentage of patients diagnosed with TD using AIMS and DSM-5 were $33 \%$ and $34 \%$, respectively. The sensitivity and specificity of AIMS in detection of TD were $67 \%$ and $83.4 \%$ respectively. The degree of agreement between the AIMS and DSM5 indicated moderate criterion validity (Cohen's kappa 0.5 ). For different raters, the agreement with the psychiatrist's diagnosis (kappa) ranged from 0.41 (CPN) to 0.63 (registrar), indicating modest reliability between raters.

\section{Conclusions}

Our findings provide preliminary evidence for the validity of AIMS in detecting TD among Sinhalaspeaking patients, when administered by a range of mental health professionals.

Key words: tardive dyskinesia, abnormal involuntary movement scale

SL J Psychiatry 2021; 12(1): 25-30

\section{Background}

The Abnormal Involuntary Movement Scale (AIMS) is designed to assess tardive dyskinesia (TD), a delayedonset iatrogenic movement disorder first described in 1964 by Faurbye (1-4). TD has been defined as involuntary athetoid or choreiform movements developing in association with the use of a dopamine antagonist, such as an antipsychotic, for at least a few months (5). The abnormal movements in TD mainly involve the tongue, lower face, jaw and extremities $(5,6)$. Characteristic manifestations include lip smacking, chewing movements, tongue protrusions, pill rolling and piano playing movements of the hand, and pelvic thrusting (7).
Recent changes in nomenclature have led to a new term, 'tardive syndromes', to serve as an umbrella term for all tardive movement disorders, while reserving the term 'tardive dyskinesia' for the classic oro-bucco-lingual choreiform movements $(8,9)$. Other tardive syndromes include tardive dystonia, tardive akathisia, tardive stereotypies and neuroleptic withdrawal emergent dyskinesia (8).

The pathogenesis of TD remains poorly understood. Dopamine receptor super-sensitivity following chronic blockade of dopamine receptors is the most favoured hypothesis $(9,10)$. However, this theory has certain 
limitations, and many alternative hypotheses related to oxidative stress, GABA neurotransmission and maladaptive synaptic plasticity, have also been proposed $(8,9)$.

The reported prevalence of TD among patients on long-term antipsychotics ranges from $20 \%$ to $50 \%$ (11). A recent meta-analysis revealed a prevalence of $20 \%$ in patients on second generation antipsychotics (SGAs), and $30 \%$ in patients on first generation antipsychotics (FGAs) (12). The annual incidence rates have been reported to be $3 \%$ with SGAs versus $7.7 \%$ with FGAs (13). These observations support the claim that SGAs cause TD less frequently than FGAs. However, if prescription of SGAs continues to increase compared to FGA use, we may see a an increased number of SGAtreated patients developing TD in future, albeit lower in proportion compared to FGA treated patients of the past $(1,8)$.

Given the high prevalence and the potentially disabling nature of TD, it is crucial to regularly screen patients on long-term antipsychotics for dyskinesia $(1,14)$. Since the AIMS was introduced in 1976 by the National Institute of Mental Health in the United States, it has been used widely in both clinical and research settings (1-3). It has been recommended to monitor for TD at least every 4 months for FGAs, every 6 months for SGAs, and every 9 months for clozapine (15). It has been estimated that TD disappears in $50 \%$ of patients after discontinuation of the offending drug (6).

AIMS is a clinician-administered scale, where abnormal movements of different body regions are evaluated $(1,2)$. It has been shown to have adequate validity and reliability in the assessment of $\mathrm{TD}$, and can be administered by any healthcare professional who has been adequately trained $(1,16,17)$. The AIMS has been previously adapted and validated in other languages $(17,18)$.

In line with worldwide trends of adopting a multidisciplinary approach in managing psychiatric patients, we in Sri Lanka are now training community psychiatry nurses (CPNs), occupational therapists and psychiatric social workers (PSWs) to play a more active role in the follow-up care of patients. The availability of a translated and validated tool will facilitate the training of nonmedical clinicians in the detection of TD in the outpatient department and the community level, so that a TD screening programme involving both medical and nonmedical clinicians can be implemented. Thus, the aim of this study was to translate the AIMS into Sinhala and to validate its use for the local setting when administered by a range of medical and non-medical professionals.

\section{Methods}

\section{Study design and setting}

As the first step we conducted an observational study to translate and pilot test the Sinhala version of the AIMS, which was thereafter validated among Sinhala-speaking patients. Data were collected at the outpatient Psychiatry Unit of North Colombo Teaching Hospital (NCTH), Ragama.

\section{Study population}

All registered outpatients attending the psychiatry clinic of NCTH, Ragama, who were on treatment with one or more antipsychotics for a minimum of one-year duration, constituted the study population. Only those who gave written informed consent were included in the study. Patients with other neurological disorders were excluded. The sample size calculation was performed by including 10 participants per item of the questionnaire (19). As there were 12 questions in the scale, this required 120 patients. To overcome non-response bias, a total sample of 132 patients was deemed adequate. Of this total, 20\% $(n=27)$ were required for the pilot test. Participants in the pilot test were not included in the validation.

\section{The Abnormal Involuntary Movement Scale (AIMS)}

The AIMS comprises 12 items, assessing abnormal movements of seven body areas, and a global judgment of severity of movements, incapacitation and patient's awareness (3). The last two items of the scale inquire regarding problems of teeth and dentures, as such problems can lead to misdiagnosis. The items are rated on a five-point scale of severity from 0 -4, i.e. 0 (none), 1 (minimal), 2 (mild), 3 (moderate) and 4 (severe). A score of 2 ('mild') in at least two items, or a score of 3 or 4 ('moderate' or 'severe') in at least one item, amounts to the presence of TD. Additionally, a total AIMS score can be calculated by summing the scores on items 1 through 7, the maximum score possible being 28 .

The AIMS is a non-proprietary scale available in the public domain. Instructions for its administration are provided with the scale. The AIMS assessment takes 10-15 minutes per patient. It involves observation of the patient in both seated and standing positions, while instructed to perform several tasks in a methodical manner. Activation procedures are used to unmask concealed dyskinetic movements.

\section{Translation and pilot testing}

Forward translation of AIMS, as well as instructions for administration, from English to Sinhala, was performed by two independent bilingual experts, and then independently back-translated into English by two other 
bilingual translators. An expert panel consisting of two consultant psychiatrists, a methodologist, and the translators was convened. Discrepancies between translations were resolved. The translated questionnaire was then pilot-tested on 27 patients to examine for comprehensibility, and feasibility of administration. A 3-day training was conducted for the raters by a consultant psychiatrist, using online educational videos and consenting real-time patients with TD. Thereafter, the raters individually assessed patients using the AIMS. At the end of the pilot test, the expert panel was reconvened to finalize the questionnaire.

\section{Validation of the Sinhala version of AIMS}

The translated scale was administered to a convenient sample of consenting outpatients attending the NCTH. Each patient was examined separately by one rater (using the AIMS) and by a psychiatrist (using DSM-5 criteria). Five raters - a medical officer, two postgraduate trainees in psychiatry, a CPN and a PSW - collected data after receiving the 3-day training on using the AIMS; each assessed a minimum of 27 patients, giving a final total of 137 patients. The psychiatrist, who was blind to the AIMS score, made the gold-standard diagnosis based on DSM5. Out of the total sample, a convenient sub-sample of 27 participants (about $20 \%$ of the total), were invited and consented to participate in a re-test planned two weeks later, however, due to the outbreak of COVID-19 and the disruption of clinics, the re-test could not be completed successfully. An interviewer-administered questionnaire was used to collect relevant sociodemographic and clinical data.

\section{Ethics}

The study received ethical approved from the Ethics Review Committee of the Faculty of Medicine, University of Kelaniya. Written informed consent was obtained from participants prior to inclusion in the study.

\section{Statistical analysis}

The data were analysed using IBM SPSS Version 20. Descriptive statistics were used to describe the sample. Sensitivity, specificity, positive and negative predictive values in detecting TD were calculated for the total sample and for each interviewer. Cohen's kappa was used to assess criterion validity, in reference to the psychiatrist's diagnosis. Cohen's kappa is an indicator of agreement between two binary outcomes, and can range from -1 to +1 . Comparison of the kappa values for different raters was used as a measure of inter-rater reliability. The total AIMS score and other global judgement scores were compared between those with TD and without, using the Mann Whitney U test.

\section{Results \\ Population characteristics}

One hundred and thirty-seven patients, receiving longterm antipsychotic medication, participated in the study. Of them, $53.3 \%(n=73)$ were male. The age ranged from 18 to 75 years, with a mean of 49 years $(S D=12.5)$.

The most common diagonsis among the participants were schizophrenia ( $\mathrm{n}=100,72 \%)$, bipolar affective disorder $(n=17,12.4 \%)$, schizoaffective disorder $(n=5,3.6 \%)$ and recurrent depressive disorder $(n=12,8.7 \%)$. The average duration of antipsychotic therapy among the patients was 14 years $(\mathrm{SD}=11.4)$. Forty-five $(32.8 \%)$ patients were taking a FGA, whereas 103 (75.1\%) patients were prescribed a SGA. Fifteen (10.9\%) patients were prescribed clozapine. Fifty-two (37.9\%) patients were on a long-acting injectable antipsychotic. Seventy-three patients (53.2\%) were on a single antipsychotic, 55 $(40.8 \%)$ were on dual antipsychotics, and the others (6.5\%) were given a combination of more than two antipsychotics. Ninety-six patients (70\%) were coprescribed an anticholinergic.

\section{Clinical utility and validity of the AIMS}

Based on the AIMS alone, 45 (32.8\%) patients were positive for TD. A total of $34.3 \%(n=47)$ patients were diagnosed with TD using DSM-5 criteria alone, by a psychiatrist. However, a note made regarding the presence of TD could be found in previous clinic notes in only 6 (12.7\%) out of 47 patients diagnosed with TD. Among those with TD, 30 patients (63.8\%) were currently on an anticholinergic.

The sensitivity and specificity of the AIMS in detecting TD, against the gold-standard diagnosis made using DSM-5 criteria, were $67 \%$ and $84 \%$ respectively. Positive and negative predictive values of the scale were $67 \%$ and $86.7 \%$, respectively. The likelihood ratio was 4.2. A Cohen's kappa of 0.5 indicated a moderate strength of agreement between AIMS and the consultant psychiatrist's DSM-5-based diagnosis, demonstrating moderate criterion validity.

The mean total AIMS score in those diagnosed with TD and those without were $9.3(\mathrm{SD}=4.5)$ and $1.5(\mathrm{SD}=1.6)$, respectively. This difference was highly significant ( $U=77$, mean rank 86 vs. 36, $\mathrm{p}<0.001$ ). Scores for global judgment of severity of movements ( $U=267$, mean rank 80 vs. 38, $\mathrm{p}<0.001$ ), incapacitation ( $\mathrm{U}=509$, mean rank 75 vs. 42, $\mathrm{p}<0.001$ ) and patient's awareness ( $\mathrm{U}=829,66 \mathrm{vs.47}$, $\mathrm{p}<0.001$ ) were significantly higher in those with TD.

Inter-rater differences

Table 1 summarizes the accuracy indicators of the AIMS, when calculated separately for the individual raters. Among the different raters, the sensitivity in detecting TD ranged from $50 \%$ to $90 \%$ and the specificity from 
Table 1. Inter-rater differences in accuracy indices of the Sinhala version of the Abnormal Involuntary Movement Scale

\begin{tabular}{|l|c|c|c|c|c|}
\hline Interviewer & Sensitivity \% & Specificity \% & PPV \% & NPV \% & Kappa \\
\hline PG trainee 1 & 50 & 94.4 & 83.3 & 77.3 & 0.49 \\
PG trainee 2 & 50 & 100 & 100 & 92 & 0.63 \\
Medical officer & 90 & 58.8 & 56.2 & 90.9 & 0.44 \\
CPN & 55.5 & 83.3 & 58.8 & 81.4 & 0.43 \\
PSW & 71.4 & 71.4 & 51.7 & 85.3 & 0.40 \\
\hline
\end{tabular}

Note: $\mathrm{PPV}=$ positive predictive value; $\mathrm{NPV}=$ negative predictive value $\mathrm{PG}=$ postgraduate;

$\mathrm{CPN}=$ community psychiatry nurse; PSW = psychiatry social worker

$58.8 \%$ to $100 \%$. Cohen's kappa values ranged from 0.4 in the PSW to 0.63 in one of the postgraduate trainees. A kappa statistic of 0.4 to 0.6 indicates a moderate strength of agreement, whereas a value of 0.6-0.8 indicates substantial agreement (20).

\section{Discussion}

This study explored the validity of a Sinhalese version of AIMS, a scale widely used in screening and monitoring TD (1). TD is an adverse effect of long-term dopamine antagonist use, with a high prevalence of $20-50 \%$ and negative effects on quality of life and prognosis among patients with psychotic illnesses $(1,8,14)$. The most appropriate strategy in managing TD is early identification and discontinuation of the offending medications $(6,15)$. Hence, a valid instrument to screen patients for TD is a necessary tool in the armamentarium of psychiatric services. Our findings indicate that approximately 2 out of 3 patients with TD can be detected by non-specialist mental health professionals using AIMS.

As evidenced by the small proportion of patients (12.7\%) in whom a diagnosis of TD had been recorded in previous clinic notes, TD is a grossly neglected adverse effect among psychiatric patients. In the busy clinic settings in Sri Lanka, it is difficult for medical officers to properly screen patients for TD. As a viable solution, non-medical healthcare professionals such as CPNs, occupational therapists and PSWs, who are now being trained and increasingly involved in the long-term care of psychiatric patients, can be included in a TD-screening programme. The availability of a Sinhala version of AIMS will facilitate the training of such personnel. Our findings attest to the validity of this scale when administered by a CPN and PSW, after a brief training programme. At present, many centres around the island conduct homevisit programmes to administer long-acting injectable antipsychotics to patients with poor treatment adherence. If the CPNs and PSWs who play a key role in community services are provided training, many cases of TD, which would otherwise be overlooked, can be detected, and intervention can be provided.

The clinical experience of the interviewer is linked to the accuracy of AIMS administration $(1,16)$. This is confirmed in our study, as postgraduate trainees in psychiatry showed greater agreement with the psychiatrist's diagnosis, compared with the CPN and PSW. The overall sensitivity of the test being limited to $67 \%$ is also a likely reflection of this. In this study, the AIMS raters were given a brief training on the administration of the scale. Therefore, if the AIMS is to be administered by nonmedical clinicians in the future with comparable accuracy, we suggest that such clinicians receive relevant training, preferably from a consultant psychiatrist, before administering the scale.

Some patients who were not diagnosed with TD by the psychiatrist were detected to have TD using AIMS. Interviewers noted that in several patients, dyskinetic movements became apparent only when 'activation procedures' were employed. When administering the AIMS, the patient is asked to tap the thumb with each finger as rapidly as possible for 10-15 seconds, while the interviewer observes the face and other areas for the appearance of abnormal movements. In the past, movements which appeared only upon activation were scored one point less than spontaneous movements (3). However, it is now recommended that activated movements be given equal significance $(1,21)$.

The total AIMS score was markedly higher in TD patients compared to others. However, this total AIMS score is not considered a linear scale, and cut-offs for diagnosing TD are not recommended $(1,3,16)$. This is because, for instance, a severe movement abnormality in one body 
area is likely to be clinically more important than mild abnormalities in multiple areas. Hence, the receiver operator characteristics analysis was not applicable. The total AIMS score is however, useful in research to evaluate the effectiveness of interventions for TD (1).

A high prevalence of TD among Sri Lankan patients is implied by the detection of TD in $34.3 \%$ of patients. This is compatible with international epidemiological data (11). As no conscious attempts were made to select patients with obvious movement disorders, and only the criterion of being on antipsychotics for a minimum of one year was used, the sample is generally representative, and the prevalence finding has some degree of validity, although limited by not being a random sample. However, a formal prevalence study would require a larger sample size.

Anticholinergics are known to exacerbate TD, and therefore, should be stopped if a patient develops TD $(6,7)$. However, in this study, many patients with TD were on anticholinergics. This further highlights the importance of screening for TD in patients who receive long-term antipsychotics.

\section{Limitations}

One of the major limitations of the study was the small sample size, and the study being confined to a single hospital in Sri Lanka. As each patient was assessed using the AIMS administered by a single rater, inter-rater reliability, in its strictest sense, could not be ascertained, and only an indirect indication of inter-rater reliability was demonstrated. An objective evaluation of the relationship between clinical experience and accuracy of TD diagnosis was not undertaken. Test-retest reliability also could not be tested, as many patients did not return for the re-test evaluation, owing to the COVID-19 related disruption in clinics.

\section{Conclusions}

This study demonstrates moderate evidence of the validity of a Sinhalese version of AIMS, as administered by a range of mental health professionals. Hence, screening for TD using the AIMS, carried out by medical and non-medical healthcare professionals could be a viable strategy to increase the detection and thereby reduce the burden of TD among psychiatric patients in Sri Lanka.

\section{Acknowledgements}

The authors would like to acknowledge the vital contributions made by Mrs. Shammi Weerasinghe (community psychiatry nurse) and Mrs. Ishani Kumari (psychiatry social worker) as raters.

\section{Statement of Contribution}

KALAK and AH developed the concept. KALAK, AH, TG, MP and AKABB developed the proposal. AKABB, MLH and TR collected data. AKABB, AH and MP analysed the data. AKABB and AH wrote the manuscript. All authors have approved the final version.

\section{Conflicts of interest}

None declared.

A K A B Baminiwatta, T Gunesekara, K A L A Kuruppuarachchi, A Hapangama, Department of Psychiatry, Faculty of Medicine, University of Kelaniya, Sri Lanka

M L Harshini, T R Bandara, North Colombo Teaching Hospital, Ragama, Sri Lanka

K M N Perera, Department of Public Health, Faculty of Medicine, University of Kelaniya, Sri Lanka

Corresponding author: AKAB Baminiwatta

Email: baminiwatta@kln.ac.lk

http://orcid.org/0000-0002-5495-2029

\section{References}

1. Kane JM, Correll CU, Nierenberg AA, Caroff SN, Sajatovic M. Revisiting the Abnormal Involuntary Movement Scale: Proceedings From the Tardive Dyskinesia Assessment Workshop, J Clin Psychiatry. 2018 May/June; 79 (3): 17cs11959

2. Menzies V, Farrell SP. Schizophrenia, Tardive Dyskinesia, and the Abnormal Involuntary Movement Scale (AIMS). J Am Psychiatr Nurses Assoc [Internet]. 2002 Apr 1;8(2):51-6. Available from: https://doi.org/10.1067/ mpn.2002.124918

3. Guy W. Abnormal Involuntary Movement Scale. In: ECDEU Assessment Manual for Psychopharmacology. 1976. p. 534-37.

4. Macaluso, Matthew Flynn, Alexandra Preskorn SH. Tardive Dyskinesia: A Historical Perspective. J Psychiatr Pract [Internet]. 2017;23(2). Available from: https:// journals.lww.com/practicalpsychiatry/Fulltext/2017/ 03000/Tardive_Dyskinesia__A_Historical_ Perspective.6.aspx

5. American Psychiatric Association. Diagnostic and statistical manual of mental disorders. 5th ed. Washington DC; 2013.

6. Harrison P, Cowen P, Burns T, Fazel M. Shorter Oxford Textbook of Psychiatry. 7th edition. 2017.

7. David M. Taylor, Thomas R.E. Barnes AHY. The Maudsley Prescribing Guidelines in Psychiatry. 13th ed. 2018. 90-92 p. 
8. Mulroy E, Balint B, Bhatia KP. Tardive syndromes. Pract Neurol [Internet]. 2020 Oct 1;20(5):368 LP - 376. Available from: http://pn.bmj.com/content/20/5/368.abstract

9. Waln O, Jankovic J. An update on tardive dyskinesia: from phenomenology to treatment. Tremor Other Hyperkinet Mov (N Y) [Internet]. 2013 Jul 12;3:tre-03161-4138-1. Available from: https://pubmed. ncbi.nlm.nih. gov/23858394

10. Teo J, Edwards M, Bhatia K. Tardive dyskinesia is caused by maladaptive synaptic plasticity: A hypothesis. Mov Disord 2012; 27: 1205-15.

11. D’Abreu A, Akbar U, Friedman JH. Tardive dyskinesia: Epidemiology. J Neurol Sci 2018; 389: 17-20.

12. Carbon M, Hsieh C-H, Kane JM, Correll CU. Tardive Dyskinesia Prevalence in the Period of Second-Generation Antipsychotic Use: A Meta-Analysis. J Clin Psychiatry 2017; 78(3): e264-78.

13. Correll CU, Schenk EM. Tardive dyskinesia and new antipsychotics. Curr Opin Psychiatry 2008; 21(2): 151-6.

14. Browne S, Roe M, Lane A, Gervin M, Morris M, Kinsella A, et al. Quality of life in schizophrenia: relationship to sociodemographic factors, symptomatology and tardive dyskinesia. Acta Psychiatr Scand 1996; 94(2): 118-24.

15. McEvoy J P, Scheifler P L, Frances A. Treatment of schizophrenia 1999. The expert consensus guideline series. J Clin Psychiatry 1999; 60(1): 3-80.
16. Lane RD, Glazer WM, Hansen TE, Berman WH, Kramer SI. Assessment of tardive dyskinesia using the Abnormal Involuntary Movement Scale. J Nerv Ment Dis 1985; 173(6): 353-7.

17. Tonelli H, Tonelli D, Poiani GR, Vital MABF, Andreatini R. Reliability and clinical utility of a Portuguese version of the Abnormal Involuntary Movements Scale (AIMS) for tardive dyskinesia in Brazilian patients. Braz J Med Biol Res 2003; 36(4): 511-4.

18. Buhmann C, Rizos A, Emmans D, Jost WH. [Intercultural adaptation of the AIMS in German language: A scale for abnormal involuntary movements]. Nervenarzt 2016; 87(4): 411-7.

19. Anthoine E, Moret L, Regnault A, Sébille V, Hardouin JB. Sample size used to validate a scale: a review of publications on newly-developed patient reported outcomes measures. Health Qual Life Outcomes [Internet] 2014 Dec 9;12:176. Available from: https://pubmed. cbi.nlm.nih.gov/25492701

20. McHugh ML. Interrater reliability: the kappa statistic. Biochem medica [Internet]. 2012; 22(3): 276-82. Available from: https://pubmed.ncbi.nlm.nih.gov/23092060

21. Munetz MR, Benjamin S. How to examine patients using the Abnormal Involuntary Movement Scale. Hosp Community Psychiatry 1988; 39(11): 1172-7. 